\title{
Fieber plus Exanthem: Masern oder Kawasaki?
}

autveränderungen, oft in Kombination mit Fieber, sind in der pädiatrischen Praxis häufig. In einer französischen Erhebung lagen diese bei $9 \%$ von 20.652 Kindern, die innerhalb von zwölf Monaten behandelt worden waren. Das mittlere Alter der Kinder betrug 4,1 Jahre. $\mathrm{Zu}$ den fieberhaften Erkrankungen, die mit einem Exanthem einhergehen, gehört das Kawasaki-Syndrom, das in der Initialphase von Masern nur schwer abzugrenzen ist. Anhand des polymorphen Exanthems allein lässt sich die Diagnose „Kawasaki“ nicht stellen. Deshalb gilt es auf zusätzliche Symptome zu achten, wie Konjunktivitis und Desquamation. Bei zwei Dritteln der Kinder liegt eine Windeldermatitis vor. Wichtig ist laut PD Dr. Hagen Ott aus Hannover der Blick auf Fußsohlen und Handinnenflächen. Denn bei Kawasaki findet sich immer eine palmare oder plantare
Beteiligung. „Es gibt wenig Krankheiten bei Kindern mit palmarer Beteiligung“, sagte Ott. Bei Masern lohnt der Blick in den Mund. Denn im Prodromalstadium treten neben Fieber und Konjunktivitiden auch Koplik-Flecken auf, also punktförmige Flecken mit einem weißen Zentrum an der Wangenschleimhaut. Ein feinfleckiges makulopapulöses Exanthem, das samt- oder sandpapierartig ist, ist typisch für Scharlach, vor allem in Kombination mit perioraler Blässe und der typischen Himbeerzunge.

Immer auf die Intensivstation gehören Kinder mit einer 4S-Erkrankung, dem "Staphylococcal Scaled Skin Syndrome“. Staphylokokken können als Superantigene das Toxic Shock Syndrome Toxin 1 und Staphylokokken-Enterotoxine bilden. Sie verursachen einen „Zytokinsturm“, der mit einer hohen Mortalitätsrate von $30-70 \%$ einhergeht. Dia-

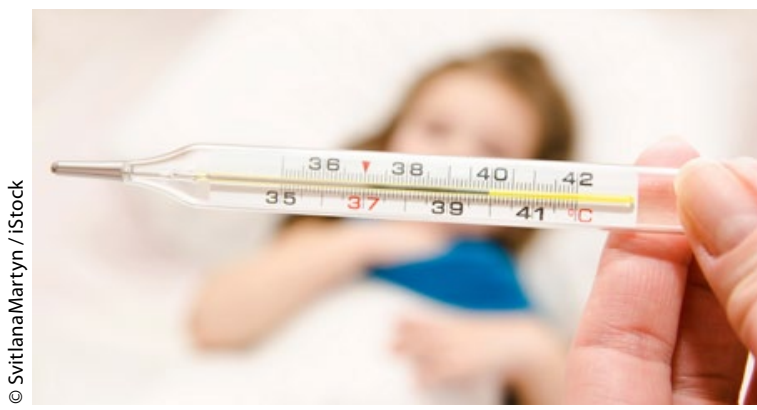

gnosekriterien sind Fieber über $38,9^{\circ} \mathrm{C}$, makulöses Exanthem mit Desquamation nach einer bis zwei Wochen, arterielle Hypotonie und eine Multiorganbeteiligung mit erhöhten CK-, Bilirubinund Kreatininwerten. Völlig harmlos, aber häufig ist dagegen die akute Urtikaria mit einer kumulativen Prävalenz von $15 \%$. Sie ist meist parainfektiös bedingt, geht nur sehr selten in eine chronische Verlaufsform über und erfordert keine weitere Diagnose. Dr. Beate Fessler

Ott H. Hautausschlag und Fieber

\section{Hier steht eine Anzeige.}

\section{丵 Springer}

